\title{
Detection of 2x2 MIMO signals
}

\author{
János Ladvánszky ${ }^{1}$
}

\begin{abstract}
In this paper, we investigate synchronization and equalization of $2 \times 2$ MIMO signals. We make a step further than that is described in our patent. In the patent, 3 PLLs and a fourchannel adaptive filter was needed. Here we decrease the number of PLLs to two and use an adaptive filter of only two channels. In addition to that, we shortly introduce the filter method and the FFT method as well, for synchronization. False detection cancellation is also mentioned. The so-called 1-bit technique has been compared to our method. After briefly introducing the ideas, detailed Matlab or AWR analyses follow. Input data are real measurements, so the analyses serve also as experimental verifications. We take a glimpse on higher order MIMO and higher order modulations as well.
\end{abstract}

Index Terms-MIMO, synchronization, equalization, false detection cancellation

\section{INTRODUCTION}

The 2 × 2 MIMO (Multiple Input Multiple Output) concept is shown in Fig. 1. The core of the idea is that 2-2 transmitter and receiver antennas can provide better system properties than two transmitter-receiver antenna pairs separately.

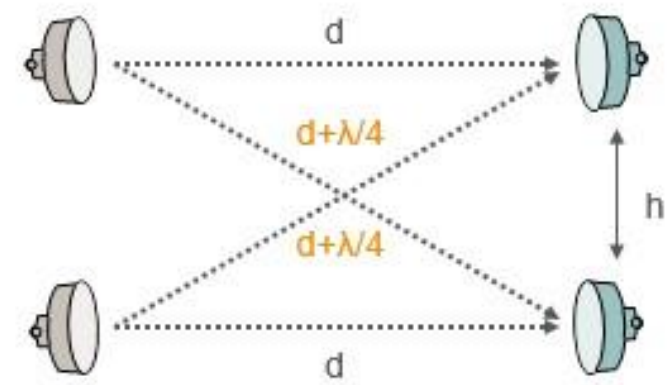

Fig. 1. The $2 \times 2$ MIMO concept. From d and the operation frequency, $\mathrm{h}$ is determined

For achieving this, a $90^{\circ}$ difference in electrical length is needed between each transmitter antenna to the two receiver antennas.

Both receiver antennas receive two signals shifted in time. If the modulation is $4 \mathrm{QAM}$, then based on the properties of the signal, the two transmitted information can be separated. For separation, estimated receiver frequencies should be known. Finding the exact receiver frequencies (synchronization) and the reconstruction of the constellation diagrams (channel equalization) can be realized by three PLLs and a four-channel adaptive filter [2].

${ }^{1}$ Formerly with Ericsson Hungary, Budapest, Hungary.

(e-mail: Ladvanszky55@t-online.hu)
In this paper, our more recent solution will be described that requires only two PLLs and a two-channel adaptive filter. The best overview about MIMO is found in [1]. In [2], our patent about MIMO is described. In [3], use of more than 4 antennas are investigated. Higher order modulations than 4QAM are discussed in [4]. DSc dissertation of the author, including a thesis about MIMO, is obtained in [5].

\section{THE MODEL}

The model of a 2 × 2 MIMO communication system is shown in Fig. 2.

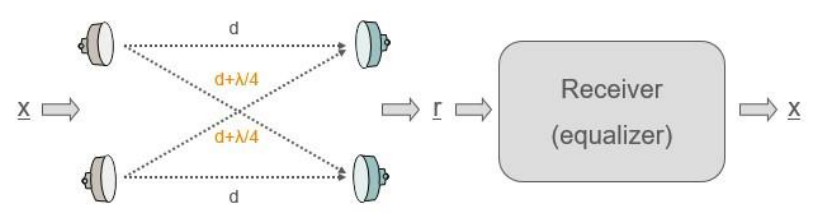

Fig. 2. Model of a $2 \times 2$ MIMO communication system. $\underline{x}, \underline{r}$ denotes the column matrices of transmitted and received signals, respectively

According to Fig. 2, an equalizer is applied for the receiver signal to reconstruct the transmitted signal.

$$
\underline{r}=\left[\begin{array}{ll}
a & b \\
b & a
\end{array}\right] \underline{x}
$$

Eq. (1) describes the antenna system. In ideal case,

$$
\begin{gathered}
a=1 \\
b=e^{-j \pi / 2}=-j
\end{gathered}
$$

And the reconstruction:

$$
\underline{\hat{x}}=\left[\begin{array}{ll}
a^{\prime} & b^{\prime} \\
b^{\prime} & a^{\prime}
\end{array}\right]^{-1} \underline{r}
$$

Our goal is

$$
|\underline{\hat{x}}-\underline{x}|=\min .
$$

And the minimum in Eq. (5) is zero if

$$
\begin{aligned}
& \underline{a}^{\prime}=\underline{a} \\
& \underline{b}^{\prime}=\underline{b}
\end{aligned}
$$

The problem is that Eq. $(6,7)$ are not fulfilled in practice. The following secondary effects may occur:

propagation loss,

wind,

mechanical tolerances,

rain,

aging,

deviation of transmitter and receiver frequencies,

time dependence of frequencies,

noise, etc. 
For that reason, both the frequency recovery and the equalizer should be adaptive. In the following, we discuss solutions for these problems.

\section{EXAMPLE}

Our work is based on a set of measured data in a file. The received signals on both antennas are measured and stored in a file. First, we try to reconstruct the frequencies. For a good start, the measurement set contains a pilot. Later, the pilot will be removed.

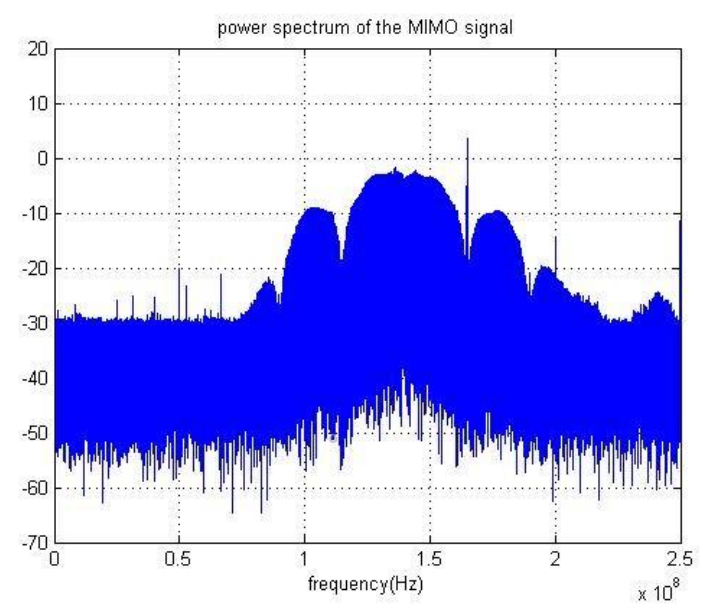

Fig. 3. FFT of the measured data with a pilot

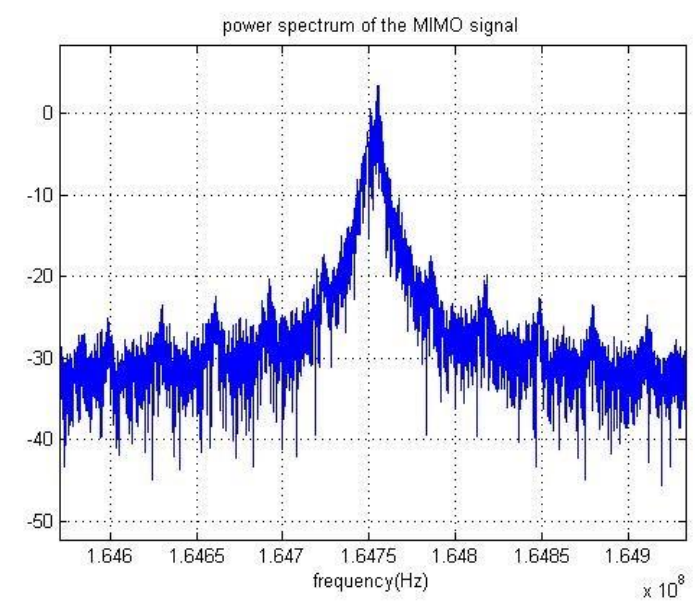

Fig. 4. The previous Figure, magnified

From Fig. 4, the pilot frequency is $164.75 \mathrm{MHz}$. But we observed that small deviations in frequency may cause a rotation of the received constellation diagram, and that may lead to false detection. For this reason, the frequencies should be determined with a high accuracy. That can be realized by a Costas loop. Matlab subsystems for the Costas loop will be described now.

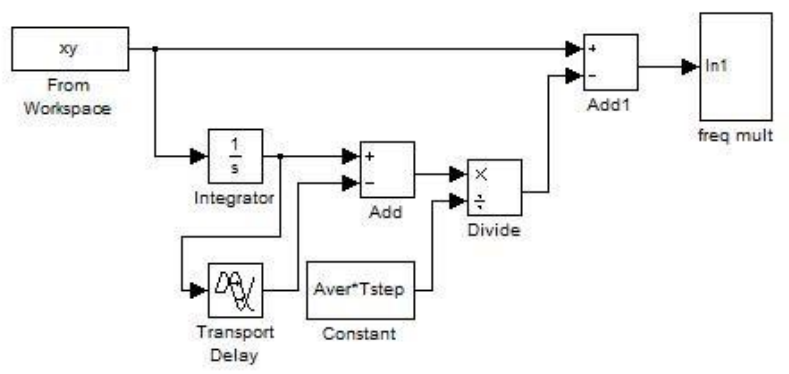

Fig. 5. The overall system

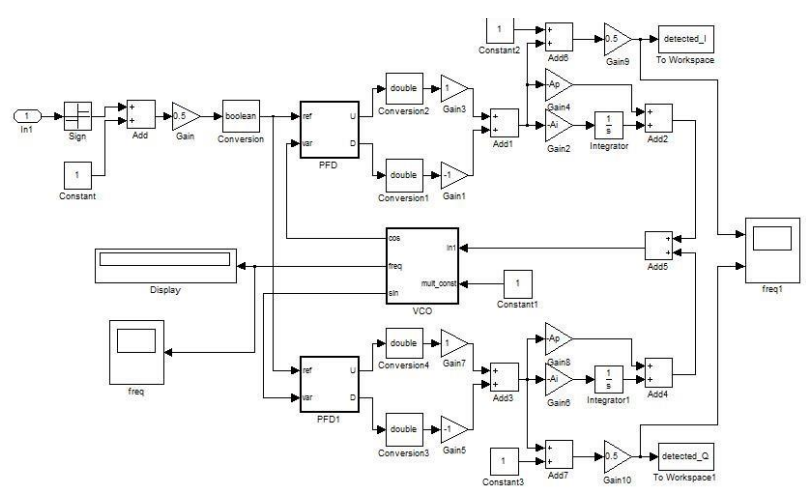

Fig. 6. The core: Costas loop. In Fig. 5, this is the block freq mult

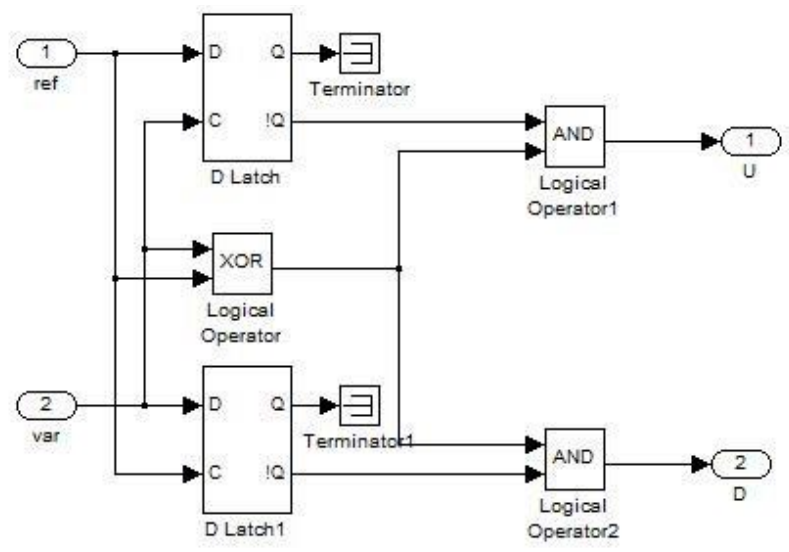

Fig. 7. The phase-frequency detector PFD in Fig. 6

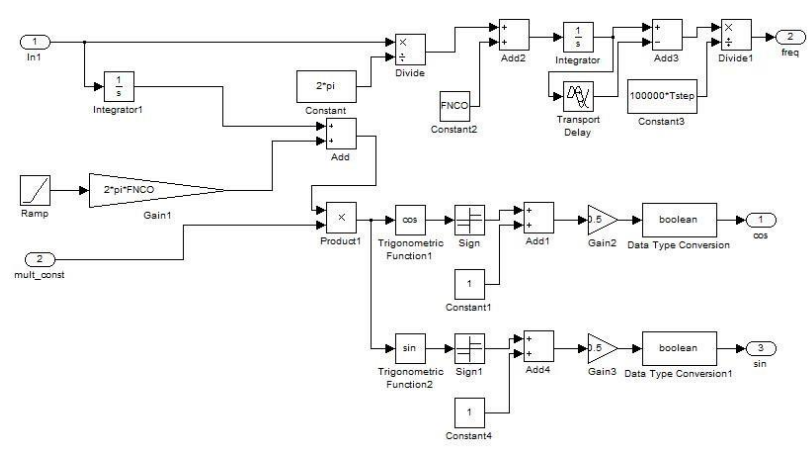

Fig. 8. The VCO in Fig. 6 
First we show how the pilot frequency is found.

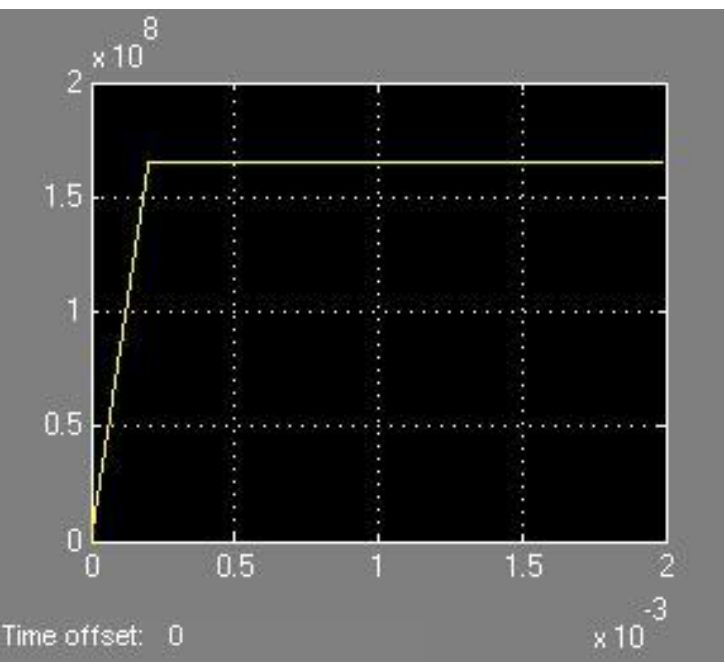

Fig. 9. Initial transient in instantaneous frequency

Settling time is about 0.2 msec. Now the detected I and Q signals are filtered.

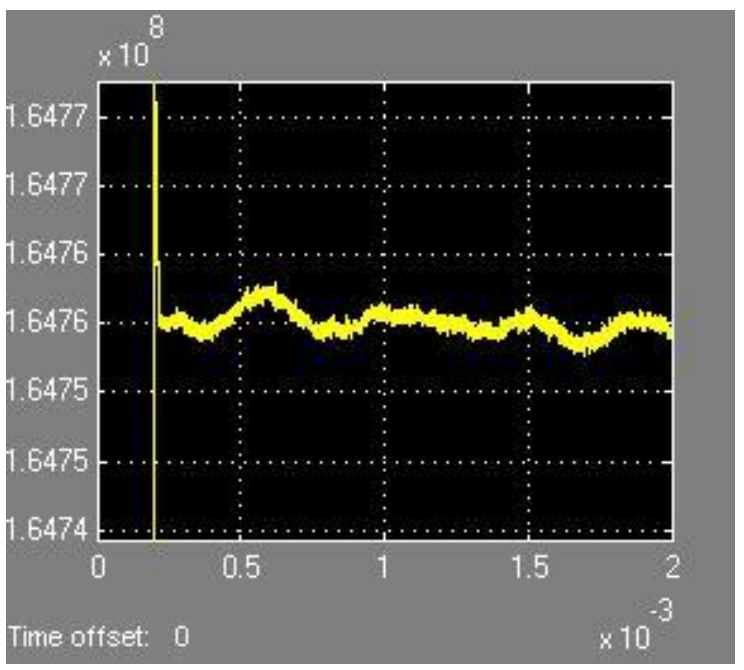

Fig. 10. The previous Figure, magnified. After settling, the frequency is kept within $1 \mathrm{kHz}$

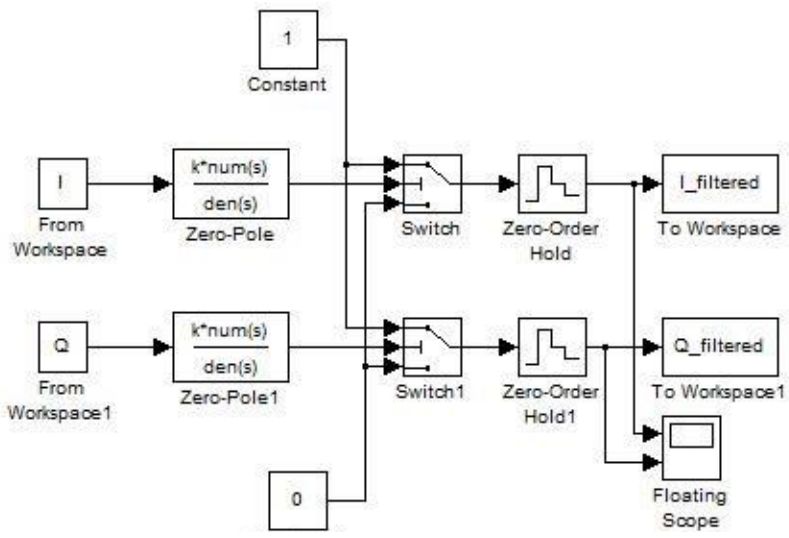

Fig. 11. Filtering system

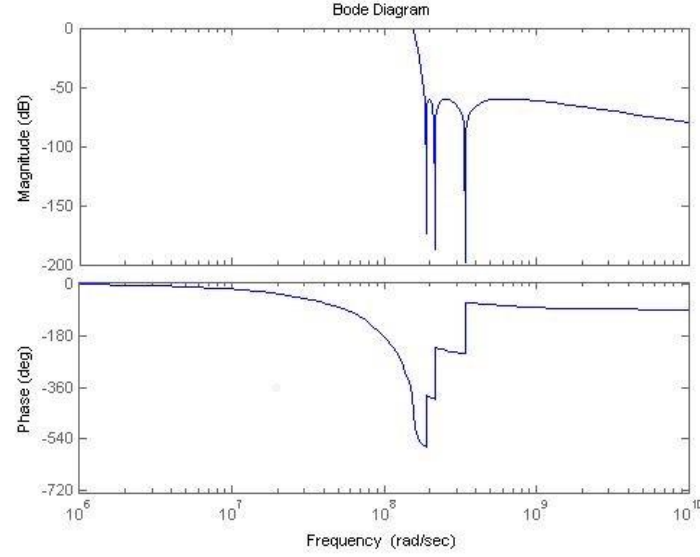

Fig. 12. Filter characteristics

As a conclusion of this example, a faster method is needed that is more accurate. Costas loop is too time-consuming.

Now we remove the pilot. The result is that the constellation diagram cannot be reconstructed in this way.

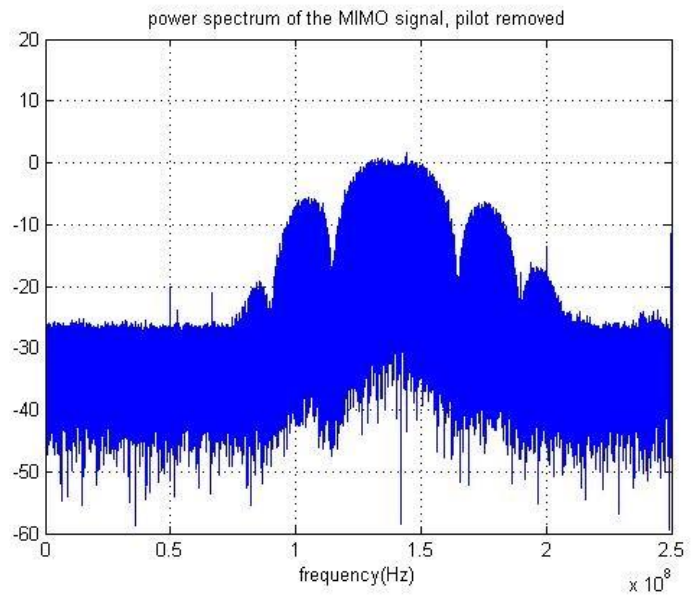

Fig. 13. FFT of the received signal without a pilot

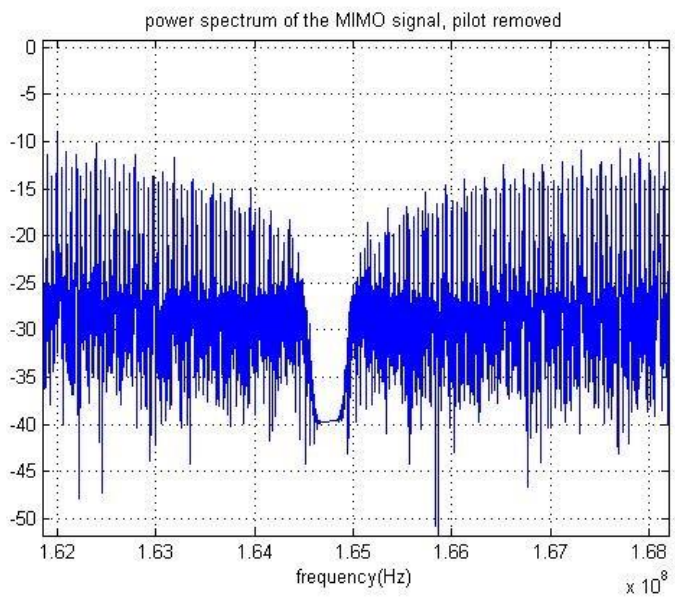

Fig. 14. The previous Figure, with increased frequency resolution 


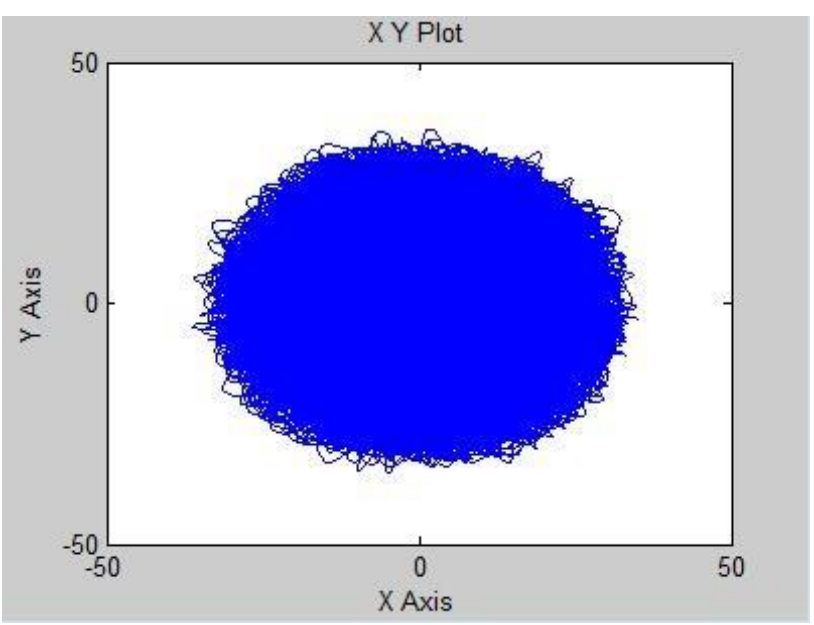

Fig. 15. The problem: The constellation diagram cannot be reconstructed in this way

\section{The MCMA EQUALIZER AND PLLS}

Basically, we have two problems. Deviation of the transmitted and received frequencies, and separation of the two transmitted signals at receiver side. Deviation is solved by PLLs, separation and signal equalization are solved by adaptive filters. Deviation can be modelled in the following way.

The baseband receiver signal $\underline{\hat{x}}$ for perfect separation, can be formulated as follows:

$$
\begin{gathered}
\underline{\hat{x}}=\underline{A}^{-1} \underline{H}^{-1} \underline{B}^{-1} \underline{r}= \\
=\left[\begin{array}{cc}
e^{-j \omega_{1} t} & 0 \\
0 & e^{-j \omega_{2} t}
\end{array}\right]\left[\begin{array}{cc}
1 / 2 & j / 2 \\
j / 2 & 1 / 2
\end{array}\right]\left[\begin{array}{cc}
e^{-j \omega_{3} t} & 0 \\
0 & e^{-j \omega_{4} t}
\end{array}\right] \underline{r}
\end{gathered}
$$

where $\omega_{1}, \omega_{2}$ are the two LO frequencies at transmitter side and $\omega_{3}, \omega_{4}$ are the two LO frequencies at receiver side. In ideal case, $\omega_{1}=-\omega_{3}$ and $\omega_{2}=-\omega_{4}$.

From our experiments we know that the equalizer can stop a slow rotation. For this reason, instead of Eq. (8), we may use

$$
\begin{gathered}
\hat{\hat{x}}=\frac{1}{c} \underline{A}^{-1} \underline{H}^{-1} c \underline{B}^{-1} \underline{r}= \\
{\left[\begin{array}{cc}
1 & 0 \\
0 & e^{-j\left(\omega_{2}-\omega_{1}\right) t}
\end{array}\right]\left[\begin{array}{cc}
1 / 2 & j / 2 \\
j / 2 & 1 / 2
\end{array}\right]\left[\begin{array}{cc}
e^{-j\left(\omega_{3}+\omega_{1}\right) t} & 0 \\
0 & e^{-j\left(\omega_{4}+\omega_{1}\right) t}
\end{array}\right] \underline{r}}
\end{gathered}
$$

where $c=e^{-j \omega_{1} t}$. Consequently, three PLLs may solve the problem. One at the output of the equalizer with frequency $\omega_{2}-\omega_{1}$, and two before the equalizer with frequencies $\omega_{3}+\omega_{1}$ and $\omega_{3}+\omega_{1}$.

According to our previous experience, the equalizer can compensate small frequency differences. It follows the three PLLs can be decreased to two. In ideal case, $\omega_{3}=-\omega_{1}$. $\omega_{2}=\omega_{1}$, and $\omega_{4}=-\omega_{2}$. Thus, with two PLLs that are locked to the input carrier frequencies, the synchronization can be solved. Details are found on Fig. 16.

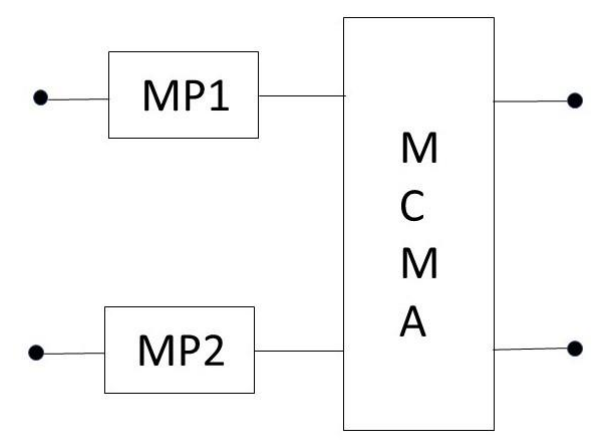

Fig. 16. Finding the proper LO frequencies at receiver side by use of two Costas loops. We use here our Costas loop version that is suggested for large noise [6]

The synchronizer consists of two Costas loops, and the VCO drive signal is formulated from both complex inputs. Realization of Fig. 16 in Matlab is presented in the next Figures that are found at the end of the paper. The detected I and Q signals are shown in Fig. 20.

Then the details of the equalizer follow. The equalizer is a four-channel adaptive filter. Inputs of the equalizer are the complex signals from the synchronizer as shown in Fig. 16.

The system diagram of the equalizer are also found at the end of the paper.

Now the I and Q signals at both channels must be reconstructed. We use the MCMA (Modified constant modulus algorithm) [1]. Its essence is the following. The equalized signal is, according to Eq. (8):

$$
\begin{aligned}
& \hat{x}_{1}=w_{1} r_{1}+w_{2} r_{2} \\
& \hat{x}_{2}=w_{3} r_{1}+w_{4} r_{2}
\end{aligned}
$$

where $w_{i}$-s are the weight factors of the adaptive filter channels. Initial values are $w_{1,1}=w_{4,1}=\frac{1}{2}, w_{2,1}=w_{3,1}=$ $\frac{1}{2} j$, according to Eq. (9). A possible choice would be $w_{1}=$ $w_{4}$ and $w_{2}=w_{3}$. This would require an ideal placement of the antennas at vertices of a rectangle. But this rectangle may be distorted by several reasons resulting in hurting condition $w_{2}=w_{3}$.

Change of the equalized signals is

$$
\begin{aligned}
& \Delta \hat{x}_{1}=\Delta w_{1} r_{1}+w_{1} \Delta r_{1}+\Delta w_{2} r_{2}+w_{2} \Delta r_{2} \\
& \Delta \hat{x}_{2}=\Delta w_{3} r_{1}+w_{3} \Delta r_{1}+\Delta w_{4} r_{2}+w_{4} \Delta r_{2}
\end{aligned}
$$

where $\Delta w_{1, k}=w_{1, k}-w_{1, k-1}$ in the kth iteration, and similarly for the other weight factors. The signals in the next iteration are $\hat{x}_{1}+\Delta \hat{x}_{1}$ and $\hat{x}_{2}+\Delta \hat{x}_{2}$. The error signals are defined as

$$
\begin{aligned}
& \varepsilon_{1}=\left\{\hat{x}_{1,1}\right\}\left(\left|\hat{x}_{1,1}\right|-R_{1}\right)+j\left\{\hat{x}_{1,2}\right\}\left(\left|\hat{x}_{1,2}\right|-R_{2}\right) \\
& \varepsilon_{2}=\left\{\hat{x}_{2,1}\right\}\left(\left|\hat{x}_{2,1}\right|-R_{1}\right)+j\left\{\hat{x}_{2,2}\right\}\left(\left|\hat{x}_{1,2}\right|-R_{2}\right)
\end{aligned}
$$

where the complex radius of the constellation diagram is $R=R_{1}+j R_{2}=|R|(1+j) / \sqrt{2}$, braces denote signum, and $r_{1}=\hat{x}_{1,1}+j \hat{x}_{1,2}$. The goal is $\varepsilon_{1}=\mathrm{min}$. and $\varepsilon_{2}=\mathrm{min}$. 
Changes of the equalized signals are

$$
\begin{aligned}
& \Delta \hat{x}_{1}=-\mu_{1} \varepsilon_{1} \\
& \Delta \hat{x}_{2}=-\mu_{2} \varepsilon_{2}
\end{aligned}
$$

where $\Delta$ denotes the change during the last sampling period. From Eq. $(12,13)$ changes of the weight factors during the last sampling period are

$$
\begin{aligned}
& {\left[\begin{array}{l}
\Delta w_{1} \\
\Delta w_{2}
\end{array}\right]=\left[\begin{array}{ll}
r_{1} & r_{2}
\end{array}\right]^{+}\left(\Delta \hat{x}_{1}-w_{1} \Delta r_{1}-w_{2} \Delta r_{2}\right)} \\
& {\left[\begin{array}{l}
\Delta w_{3} \\
\Delta w_{4}
\end{array}\right]=\left[\begin{array}{ll}
r_{1} & r_{2}
\end{array}\right]^{+}\left(\Delta \hat{x}_{2}-w_{3} \Delta r_{1}-w_{4} \Delta r_{2}\right)}
\end{aligned}
$$

in the kth iteration, where + denotes Moore-Penrose generalized inverse. Here we exploit the fact that the generalized inverse gives the minimum norm solution of an under-determined system [10].

The algorithm starts with $w_{1}, w_{2}, w_{3}, w_{4}, r_{1}$ and $r_{2}$. Then Eq. $(10,11,14-19)$ are executed. Then w-s and r-s are delayed. Similarly, for the other channel. The given algorithm significantly differs from known algorithms [1]. Robustness of the algorithm has been observed during all runs.

\section{RELATED QUESTIONS}

Here we summarize some other important ideas related to $2 \times 2$ MIMO detection.

Filter method

Essence of the filter method is that instead of a PLL, a filter is applied for synchronization. Advantage with respect to the previous Chapter is, that the random changes on LO frequencies are much smaller, and locking problems are eliminated. Hardware requirements are of the same amount.

FFT method

The filter function can be realized by applying FFT of the input signal. FFT is computation consuming, so it is a hard decision how often the FFT is made. Advantages are the same as for the filter method.

False detection cancellation

False detection occurs when the received constellation diagram suddenly rotates by $90^{\circ}$. Originally, we made a great effort on false detection cancellation. However, in our previous publication [8], we pointed out that the most efficient solution of this problem is application of differential coding. Thus, we omit here the list of other methods for false detection cancellation.

The 1-bit method [9]

The main difference between this and our method is that in this method, the signal after synchronization is digitized. This is a limiting case, in the literature few bits A/D conversion can also be found. The author's opinion is that the possible advantage of decreasing the noise effects is virtual, because it remains at the random variation of zero crossings. Moreover, strong learning algorithms may be needed for equalization that are time consuming.

\section{More than 4 antennas}

In case of more than 4 antennas, differences in distances between a transmitter antenna and neighboring receiver antennas cannot be kept constant. Even synchronization is more difficult, because there are many transmitter frequencies that can be different all. Equalization is to invert the matrix describing the relation between separate transmitter and receiver signals. But these are possible [3], however, the extra advantage of more than 4 antennas decrease.

\section{Higher order modulation}

Essentially arbitrary order (power of 2) can be used [4]. In our opinion, its significance is not too high because for higher order modulation, noise sensitivity rapidly increases, and it is a better solution to apply lower order modulation at higher speed.

\section{CONCLUSIONS}

The synchronization by two Costas loops and the equalization using an adaptive filter have been detailed. The algorithms are robust. The system can follow the random variations of any kind that are included in the measured data. We call the attention to the fact that for synchronization we used our best version of Costas loop, but without differential coding, and for equalization, we corrected the errors found in the literature. As a result, the standing constellation diagrams appear soon at the output, and remain almost constant for the rest of the measurement interval. Other Hungarian efforts concerning MIMO technique are found in [11]. A possible use of MIMO is presented in [12].

In answering to the reviewer openly, this is not a summary paper. Material in Section 4 is brand new, especially Eq. $(18,19)$. As its previous version is patented [2], it is used worldwide.

\section{ACKNOWLEDGMENTS}

This work was started at the microwave center of Ericsson in Gothenburg, Sweden in 2010 and continued at Ericsson Hungary, Budapest. The good research atmosphere at both places should be acknowledged.

The whole work in Gothenburg was performed with Dr. G. Kovács, my younger colleague at Ericsson. He was an excellent partner in every phase of the work. Special thanks for the first in-house review of this paper. Mrs. Anna Rhodin was our host in Gothenburg. She obtained the measurement data we used in our experiments. With her, three of us became the authors of our patent in this work. From management side, we were supported by Mr. V. Beskid, Dr. Á. Vámos and Dr. P. Verebély, and finally by Dr. B. Kovács. 


\section{REFERENCES}

[1] Ingason, T., Haonan, L.: "Line-of-Sight MIMO for Microwave Links", MSc Thesis, Chalmers University of Technology, Göteborg, Sweden, July 2009

[2] Ladvánszky, J., Rhodin, A., Kovács, G.: "Synchronization and equalization of $2 \times 2$ LoS MIMO communication channels", invention disclosure, Ericsson Hungary, 2010, P32542

[3] Han, D., Lee, C., Park, H., Kahng, S.: "Antenna Design for 4-by-4 MIMO Communication”, 2016 International Conference on Signal Processing and Communication (ICSC)

[4] Jacobsson, S., Durisi, G., Coldrey, M., Goldstein, T., Studer, C. "Nonlinear 1-Bit Precoding for Massive MU-MIMO with HigherOrder Modulation", 2016 50th Asilomar Conference on Signals, Systems and Computers, 6-9 Nov. 2016, Pacific Grove, CA, USA

[5] Ladvánszky, J.: "Integrated optical communication systems", DSc dissertation, Hungarian Academy of Sciences

[6] Ladvánszky, J., Kovács, B.: "Methods and apparatus for signal demodulation", Ericsson patent, PCT/SE2018/050198, 2018

[7] Kuznetsov, N. V., Blagov, M. V., Kudryashova, E. V., Ladvánszky, J. Yuldashev, M. V., Yuldashev, R. V.: "Non-linear analysis of a modified QPSK Costas loop", Elsevier, IFAC-PapersOnLine Volume 52, Issue 16, 2019, Pages 31-35

[8] Ladvánszky, J.: "A Costas loop with Differential Coding", International Journal of Contemporary Research and Review ISSN 0976 - 4852, October, 2017IVolume 08IIssue 10

[9] Mo, J., Schniter, P., and Heath JR., R. W.: "Channel Estimation in Broadband Millimeter Wave MIMO Systems with Few-Bit ADCs", ArXiv: 6 Dec. 2017

[10] Boyd, S.: "Least-norm solutions of undetermined equations", lecture, EE263 Autumn 2007-08, https://see.stanford.edu/materials/ lsoeldsee263/08-min-norm.pdf

[11] Fodor, G., Pap, L., Telek M.: "Recent Advances in Acquiring Channel State Information in Cellular MIMO Systems", Infocomm. Journal, $3 / 2019$

[12] Hilt, A.: "Microwave Hop-Length and Availability Targets for the 5G Mobile Backhaul", IEEE 42nd Telecommunications and Signal Processing Conference, TSP'2019, Budapest, Hungary, 1-3 July 2019. DoI: $10.1109 /$ TSP.2019.8768870

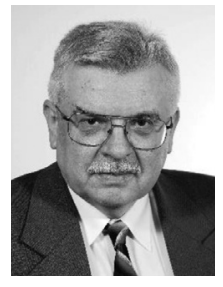

János Ladvánszky was born in Budapest, Hungary in 1955. He received his M.Sc. from the Budapest University of Technology and Economics in 1978 $\mathrm{He}$ received the $\mathrm{Ph} . \mathrm{D}$. from the Hungarian Academy of Sciences in 1988. He is now just before the defense of his D.Sc. dissertation at the Hungarian Academy of Sciences. His research interests include circuits and systems theory, with microwave and optical applications, and more recently, quantum communications. He is the World Champion in writing scientific papers in 2018, in electrical engineering. He is an evaluator for the circuits and systems analysis program AWR, and available at LinkedIn, ResearchGate and Facebook.

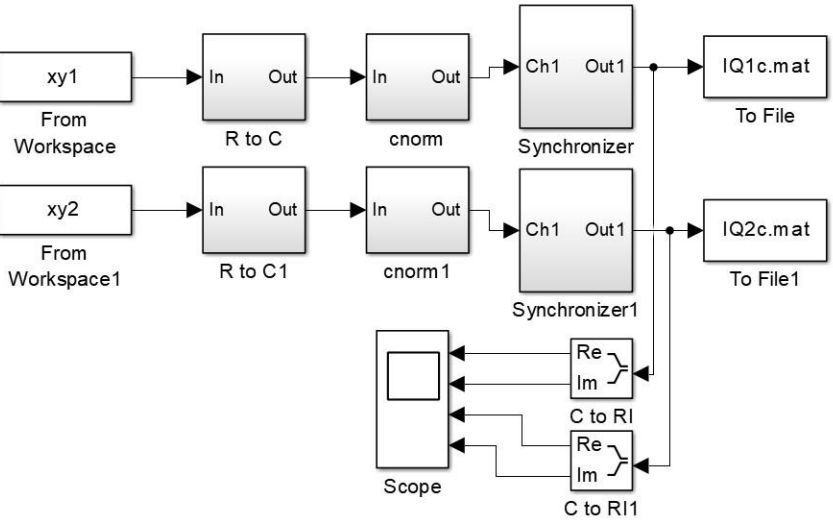

Fig. 17. The synchronization system in Matlab

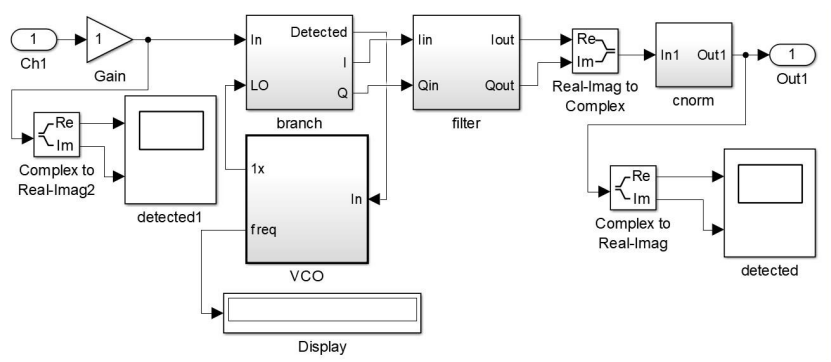

Fig. 18. The Synchronizer in Fig. 17

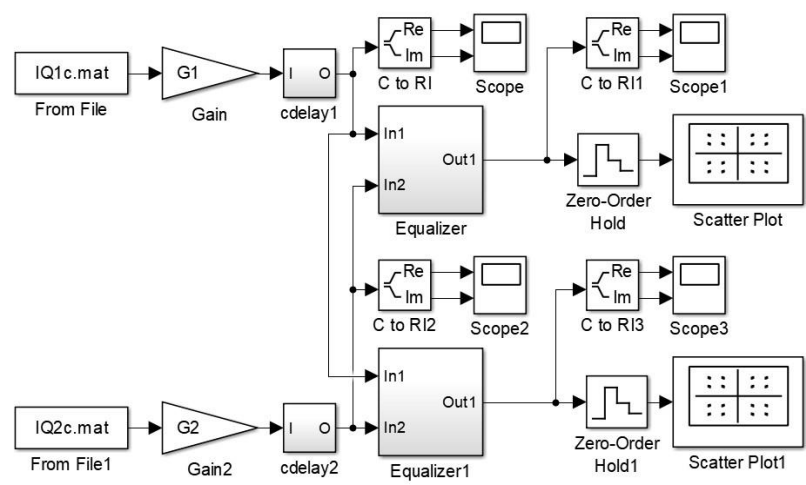

Fig. 19. The equalizer system diagram in Matlab. Input files containing data after synchronization, are IQ1c.mat and IQ2c.mat. Outputs are scatter plots of the equalized constellation diagrams 


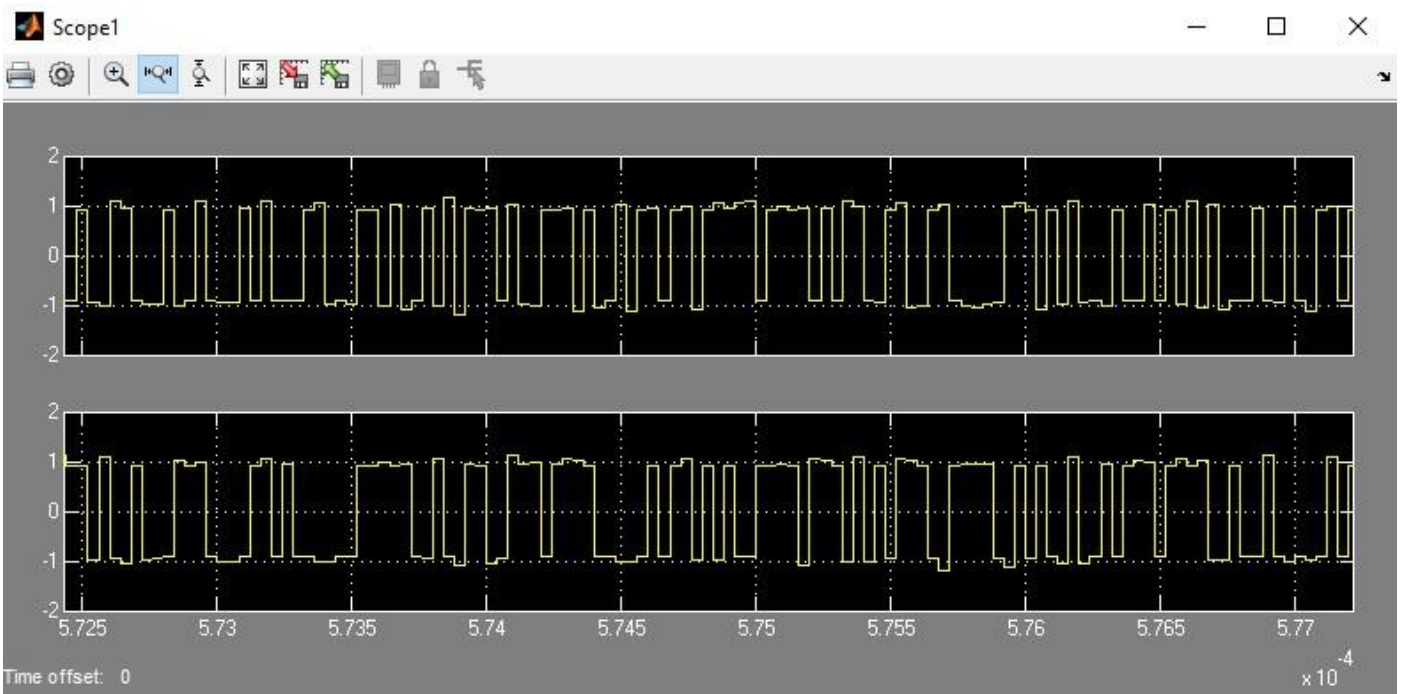

Fig. 20. Output I and Q signals in Fig. 19, Scope 1, with high time resolution

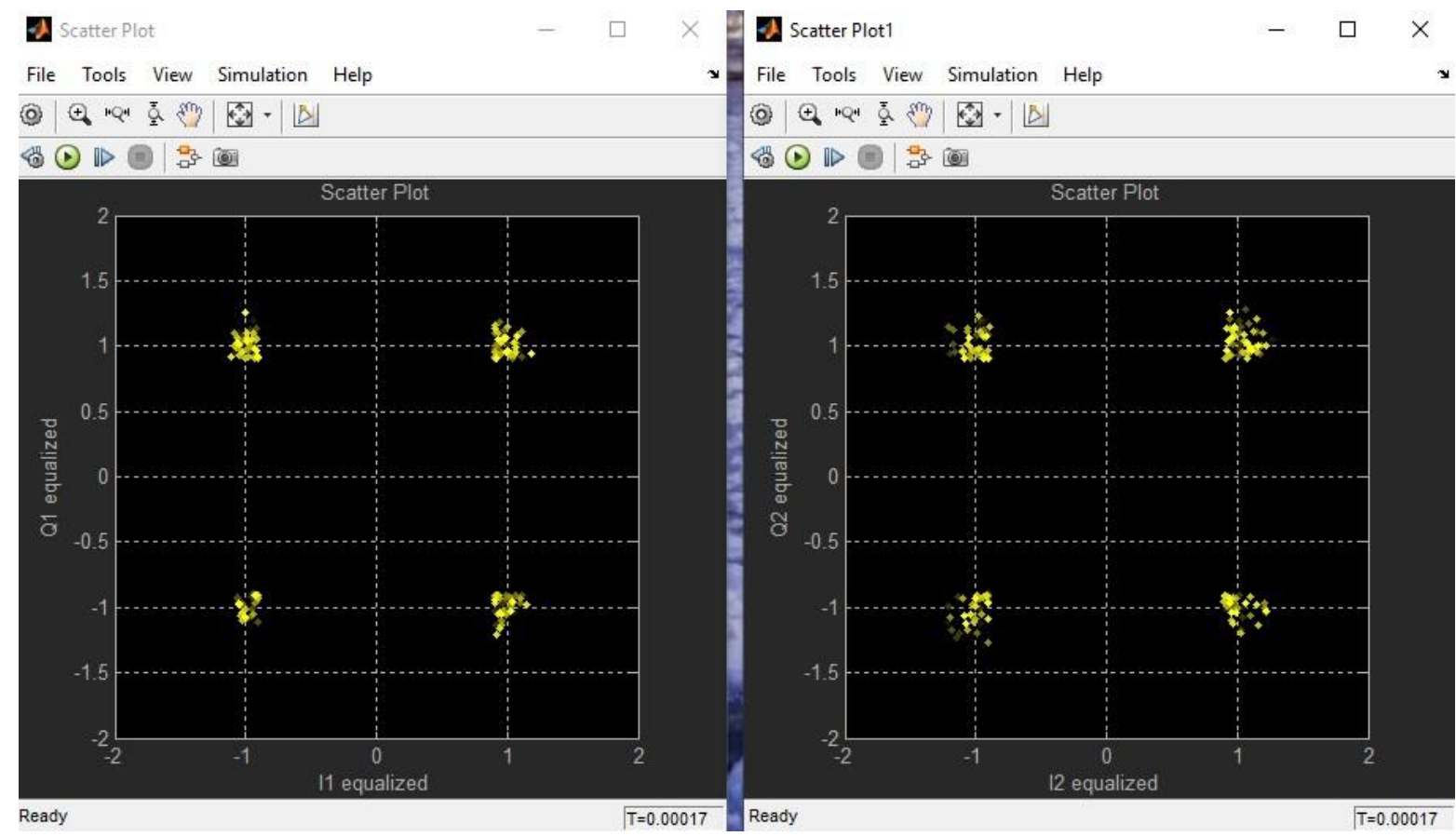

Fig. 21. Output scatter plots in Fig. 19. At the lower right corner of each scatter plot, it can be observed that these constellation diagrams were reconstructed at the very beginning of the simulation time 